\title{
Resistencia Antibiótica en Salmonella enterica serovar Typhimurium aisladas de granjas porcícolas en el departamento del Tolima*
}

\section{Antibiotic resistance in salmonella enterica serovar Typhimurium isolated from hogfarms in the department of Tolima}

\author{
Miguel A. Fierro - Amature ${ }^{1}$, Camilo A. Osorio - Amortegui ${ }^{1}$ \\ Luz C. Fandiño de Rubio², lang S. Rondón - Barragán ${ }^{1 *}$ \\ ${ }^{1}$ MVZ ** Bacterióloga "'MVZ MSc, Grupo de Investigación en Inmunología y Fisiopatología Animal, \\ Facultad de Medicina Veterinaria y Zootecnia, Universidad del Tolima \\ *Proyecto "Evaluación epidemiológica de la presencia de Salmonella spp y determinación de factores \\ de riesgo asociados a su presentación en granjas del Departamento del Tolima" Ministerio de \\ Agricultura y Desarrollo Rural (MADR) - Asociación Colombiana Porcicultores (ACP)
}

Recibido: Diciembre 7 de 2010. Aprobado: Marzo 2 de 2011

\begin{abstract}
RESUMEN
La salmonelosis es una enfermedad causada por bacterias del género Salmonella, familia Enterobacteriaceae; siendo de gran importancia en los cerdos, la presencia de Salmonella enterica serovar Typhimurium que ocasiona enterocolitis necrosante y la Salmonella choleraesuis que provoca la forma septicémica caracterizada por hepatitis, neumonía y vasculitis cerebral. El uso indebido de antibióticos, incluyendo la subdosificación así como la terapia con un único principio activo y en algunos casos la utilización de antibióticos como preventivos principalmente mediante la inclusión del mismo en el alimento ha conllevado al desarrollo de resistencia a antimicrobianos en bacterias asociadas a animales de consumo. El presente trabajo tiene por objetivo determinar la resistencia a antibióticos de la Salmonella enterica serovar Typhimurium aisladas en producciones producción porcícolas del Departamento del Tolima, en diferentes etapas del ciclo de producción.
\end{abstract}

Palabras clave: Porcinos, Resistencia antibiótica, Salmonella.

\section{ABSTRACT}

Salmonellosis is a disease caused by bacteria, genera Salmonella, famlily Enterobacteriaceae; which is important in pigs. The presence of Salmonella enterica serovar Typhimurium causes necrotizing enterocolitis and Salmonella choleraesuis causes septicemic form characterized by hepatitis, pneumonia and 
cerebral vasculitis. Incorrect use of antibiotics, including subdosifying as well as unique therapy and in other cases preventive use of antibiotics has lead to the development of antibiotic resistance. The aim of this work is assess the antibiotic resistance of Salmonella enterica serovar Typhimurium isolated from pig farms at different production steps in the Department of Tolima.

Key words: Pigs, antibiotic resistance, Salmonella.

\section{INTRODUCCIÓN}

En la actualidad, las ETAs (Enfermedades transmitidas por alimentos) representan un importante campo de estudio en la salud pública, dado que son enfermedades que resultan de la ingestión de alimentos que contienen microorganismos perjudiciales vivos o sus toxinas para el consumo humano y animal. Los alimentos corren el riesgo de contaminación durante el proceso de producción, almacenamiento y consumo, constituyendo un problema de salud pública (FAO/OMS, 2007).

En Colombia, durante el año 2007 se reportaron al Sistema Nacional de Vigilancia en Salud Pública (SIVIGILA) 5.563 casos de enfermedades transmitidas por alimentos $y$, de los únicos 5 brotes con seguimiento, 4 ocurrieron en restaurantes y 1 en el hogar (INS, 2008). No obstante, en el país existe subregistro de la notificación de casos de enfermedades transmitidas por alimentos. La Organización Mundial de la Salud plantea que la mayoría de las contaminaciones de alimentos que generan enfermedades transmitidas por alimentos ocurren por problemas sanitarios y de manipulación, dentro del hogar y en sitios en donde se elaboran para la venta (Florez et al., 2008). Como lo describe Florez et al., (2008), en un trabajo realizado en 5 ciudades de Colombia, un gran porcentaje de los factores de riesgo incluyen malas prácticas en el momento de la manipulación del

\section{MATERIALES Y MÉTODOS}

\section{Granjas}

Este estudio se realizó en el Departamento del Tolima. El tamaño de la muestra se calculó a través alimento y desconocimiento de cómo corregirlas, además, en el mismo trabajo, se demostró alta presencia de parásitos intestinales en $26,9 \% ; 49$ (3,8\%) fueron positivos para parásitos patógenos, 6 $(0,46 \%)$ para enterobacterias patógenas y $8(0,52 \%)$ cultivos de manos, para Staphylococcus aureus.

La salmonelosis es una enfermedad causada por una bacteria del género Salmonella que pertenece a la familia Enterobacteriaceae; siendo de gran importancia en los cerdos, la presencia de Salmonella enterica ser Typhimurium que ocasiona enterocolitis necrosante y la Salmonella choleraesuis que (Vadillo, 2000; Quinn et al., 2001). La ingestión es la principal ruta de infección así como la mucosa del tracto respiratorio y la conjuntiva (Quinn et al., 2001). El uso indebido de antibióticos, incluyendo la subdosificación así como la terapia con un único principio activo y en algunos casos la utilización de antibióticos como preventivo principalmente mediante la inclusión del mismo en el alimento ha conllevado al desarrollo de resistencia a antimicrobianos en bacterias asociadas a animales de consumo (Rondón et al., 2006). El presente trabajo tiene por objetivo determinar la resistencia a antibióticos de la Salmonella enterica serovar Typhimurium aisladas en producciones producción porcícolas del Departamento del Tolima, en diferentes etapas del ciclo de producción. de la fórmula descrita por Thrusfield (2007) con un nivel de confianza del $95 \%$ y una precisión del $10 \%$. Siendo entonces:

$$
n=1,96^{2} P \exp \left(1-P_{e x p}\right) / d^{2}
$$


Donde $n$ es el tamaño de muestra requerida, $P_{\exp }$ la prevalencia esperada y $d^{2}$ la precisión absoluta deseada.

La selección de las granjas se hizo por el método no probabilístico de disponibilidad y conveniencia, buscando la representatividad en el Departamento del Tolima. Los animales dentro de las mismas se escogieron completamente al azar, proporcional de acuerdo con el número de animales en la granja y discriminados por ciclos de producción.

\section{Muestreo y aislamiento de Salmonella}

Para cada etapa de producción y en producciones de ciclo completo, se tomaron muestras de heces (250 g; $n=88$ ), mediante masaje rectal de animales en la misma etapa de producción y en unidades de producción contiguas. De la misma manera, las muestras de alimento ( $250 \mathrm{~g} ; n=54$ ) fueron tomadas en comederos, mediante bolsas $\mathrm{Nasco}^{\circledR}$ estériles. Las muestras de aguas (200 ml; $n=21)$ tanto de afluentes como de efluentes se tomaron mediante recipientes de plástico esterilizados previamente y mantenidos en refrigeración hasta su procesamiento. Para el aislamiento de la Salmonella spp fueron utilizados los protocolos internacionales estándar (ISO 6579:2002; ISO 6579:2002/Amd1:2007; Reid, 2009). Brevemente, las muestras fueron incubadas en agua peptonadabufferada para su pre-enriquecimiento, con un tiempo de incubación de 24 horas a $37^{\circ} \mathrm{C}$, posterior al cual fueron dispuestas en caldo tetrationato

\section{RESULTADOS Y DISCUSIÓN}

Salmonella spp constituye uno de los agentes infecciosos más comunes y ampliamente distribuidos dentro de las enfermedades trasmitidas por los alimentos, instituyéndose en un problema de salud pública y animal. Se conoce que la prevalencia de Salmonella en granjas porcinas del departamento del Tolima es baja, cercana al 3,6 \% (Fierro et al., datos sin publicar). No se evidenciaron animales con signos clínicos compatibles con alguna forma entérica o septicémica de salmonelosis u otra enfermedad clínica subyacente. Además, los animales a los cuales les fue aislado
(Müller-Kauffmann) incubadas a $37^{\circ} \mathrm{C}$ y Rappaport Vassiliadis (incubadas a $42{ }^{\circ} \mathrm{C}$ ), para su enriquecimiento selectivo. De allí fueron sembradas en agar SS (Salmonella-Shigella) y agar XLD (Xilosa Lisina Desoxicolato). Las colonias compatibles fueron subsembradas en agar McConkey y Tripticasa Soya Agar (TSA) confirmadas como Salmonella spp, mediante desafío con anticuerpos Poli A-I + Vi (Difco $\left.{ }^{\circledR} 222641\right)$. De la misma manera fueron confirmadas bioquimícamente mediante galería API ${ }^{\circledR} 20 \mathrm{E}$ (Biomereux, Francia). Las cepas fueron enviadas al Instituto Colombiano Agropecuario - ICA para su serotipificación.

\section{Sensibilidad antibiótica}

Para la prueba de sensibilidad antimicrobiana, se analizaron mediante la técnica Kirby-Bauer (Difusión en agar), para determinar patrones de sensibilidad frente a un panel de agentes antimicrobianos (Ampicilina, Amoxicilina, Apramicina, Ciprofloxacina, Cloranfenicol, Cefalexina, Enrofloxacina, Gentamicina, Kanamicina, Lincomicina, Ácido Nalidíxico, Neomicina, Nitrofuranoina, Tetraciclina y Sulfametoxazole/Trimetropin). La suspensión bacteriana se determinó de acuerdo a la dilución 0,5 de la escala de turbidez de McFarland, utilizando el agar Mueller-Hinton (Oxoid, Alemania) como medio de siembra. El protocolo utilizado fue el descrito por la NCCLS (2005) donde además se describe la interpretación de halos de inhibición, medidos a las 24 horas de incubación a $37^{\circ} \mathrm{C}$.

el microorganismo tanto en heces como en alimento correspondían a etapas de producción finales incluyendo ceba, gestación y reproductores.

En el presente trabajo fueron aisladas 6 cepas, tanto de alimento $(n=4)$ como heces $(n=4)$, excepto de aguas efluentes o afluentes. Todas las cepas serotipificadas correspondieron a Salmonella enterica serovar Typhimurium. Solo una de las cepas, la cepa 2, presentó un comportamiento en la sensibilidad antibiótica diferentes a las restantes 5 (Tabla 1). Es de anotar que las cepas aisladas 
de alimento tuvieron todas un mismo patrón de resistencia antibiótica. Sisak et al., (2006) describen que la presencia de resistencia antibiótica en diferentes cepas y serovariedades, ratifican que la sensibilidad a los antibióticos no está restringida al serotipo Typhimurium, y dicha resistencia puede ser variable incluso en fagotipos similares.

Tabla 1. Resultados antibiograma de cepas de Salmonella

\begin{tabular}{|c|c|c|c|c|}
\hline $\begin{array}{c}\text { AGENTE } \\
\text { ANTIMICROBIANO }\end{array}$ & ABREVIATURA & CONCENTRACIÓN & $\begin{array}{r}\text { RESULTADO** } \\
\text { Cepa } 1,3,4,5,6\end{array}$ & $\begin{array}{c}\text { RESULTADO } \\
\text { Cepa } 2\end{array}$ \\
\hline ACIDO NALIDÍXICO & NA & $30 \mu \mathrm{g}$ & $\mathbf{S}$ & $\mathbf{S}$ \\
\hline AMOXICILINA & AML & $10 \mu \mathrm{g}$ & $\mathbf{S}$ & $\mathbf{S}$ \\
\hline AMPICILINA & AMP & $10 \mu \mathrm{g}$ & $\mathbf{S}$ & $\mathbf{S}$ \\
\hline APRAMICINA & APR & $15 \mu \mathrm{g}$ & $\mathbf{S}$ & $\mathbf{I}$ \\
\hline CEFALEXINA & CL & $30 \mu \mathrm{g}$ & $\mathbf{S}$ & $\mathbf{S}$ \\
\hline CIPROFLOXACINA & CIP & $5 \mu \mathrm{g}$ & $\mathbf{S}$ & $\mathbf{S}$ \\
\hline CLORANFENICOL & $\mathrm{C}$ & $30 \mu \mathrm{g}$ & $\mathbf{S}$ & $\mathbf{S}$ \\
\hline ENROFLOXACINA & ENR & $5 \mu \mathrm{g}$ & $\mathbf{S}$ & $\mathbf{S}$ \\
\hline GENTAMICINA & GM & $10 \mu \mathrm{g}$ & $\mathbf{S}$ & $\mathbf{S}$ \\
\hline KANAMYCINA & $\mathrm{K}$ & $30 \mu \mathrm{g}$ & $\mathbf{S}$ & $\mathbf{S}$ \\
\hline LINCOMICINA & MY & $2 \mu \mathrm{g}$ & $\mathbf{R}$ & $\mathbf{R}$ \\
\hline NEOMYCINA & $\mathrm{N}$ & $30 \mu \mathrm{g}$ & $\mathbf{S}$ & $\mathbf{I}$ \\
\hline NITROFURANTOINA & $\mathrm{F}$ & $300 \mu \mathrm{g}$ & $\mathbf{S}$ & $\mathbf{S}$ \\
\hline $\begin{array}{l}\text { SULFAMETOXAZOLE/ } \\
\text { TRIMETHOPRIM }\end{array}$ & SXT & $25 \mu \mathrm{g}$ & $\mathbf{S}$ & $\mathbf{S}$ \\
\hline TETRACICLINA & $\mathrm{TE}$ & $30 \mu \mathrm{g}$ & $\mathbf{R}$ & $\mathbf{R}$ \\
\hline
\end{tabular}

${ }^{*} \mathrm{~S}=$ sensible, $\mathrm{I}=$ intermedio, $\mathrm{R}=$ resistente. Cepas 1,2 = aisladas de heces; Cepas 3,4,5,6 = aisladas de alimento

Los antibiogramas dieron como resultado un bajo porcentaje de resistencia entre las cepas de Salmonella aisladas; siendo solo resistentes a Lincomicina y a Tetraciclina. Al conocimiento de los autores existen pocos reportes de evaluación de la resistencia a lincomicina en cepas de Salmonella spp. No obstante, De Geeter et al., (1976) demostraron que la adición de lincomicina a dietas de porcinos infectados con Salmonella Typhimurium no altera la diseminación de esta a través de las heces. De la misma manera, describen que la sensibilidad de la $S$. Typhimurium a otros antibióticos no fue alterada por la exposición previa a lincomicina. Thakur \& Bajaj (2006) así como Harakeh et al (2005) demostraron altos niveles de resistencia de Salmonella spp a la clindamicina la cual es una lincosamida igual a la Lincomicina y frente a la cual se ha demostrado completa reacción cruzada, por lo cual se debe analizar la posibilidad de dicha reacción en las cepas de campos aisladas. Además, como lo propone Carlsson y Ferris (2000) puede existir resistencia inducida a diferentes antibióticos en aislados de Salmonella cuando son expuestas de derivados de penicilina. Por otro lado, Arroyo y Arroyo (1995) demostraron resistencia frente a este antibiótico en 24 cepas de Salmonella aisladas.

De la misma manera, los datos observados coinciden con lo descrito por Rajic et al., (2004), Grebeyes et al., (2000), Weill (2008) y Pinto et al., (2010) quienes determinaron a la tetraciclina como el agente antimicrobiano frente al cual está desarrollada mayor resistencia. Sisak et al., (2006) demostraron un alto porcentaje de resistencia (84.7 \%) a las tetraciclinas de cepas de Salmonella Typhimurium aisladas de cerdos, tanto en plantas de sacrificio como en explotaciones porcícolas. 
De otra parte, se evidenció sensibilidad intermedia compartida por parte de las cepas aisladas frente a Neomicina, un aminoglucósido que es poco utilizado en el control y tratamiento de enfermedades entéricas en el ganado porcino en la región. Dicho hallazgo contrasta con lo encontrado por Pérez (2005) quien halló resistencia frente a Neomicina en un estudio longitudinal realizado en 2 granjas porcícolas, también se encontró sensibilidad intermedia frente Ampicilina. Este último hallazgo difiere de lo reportado por Mejía, (2003) quien encontró resistencia frente a la Ampicilina, un agente que ha sido utilizado durante décadas en la producción porcina como agente terapéutico, profiláctico y el cual tiene una resistencia altamente difundida (Breuil et al., 2000). Recientemente, Pinto et al., (2010) reportaron resistencia a ampicilina en el $81.25 \%$ de 16 cepas de Salmonella aisladas.

De la misma manera, se halló sensibilidad intermedia a la Nitrofurantoina en contraste con lo observado por Breuil et al., (2000), Martel et al., (2000) y Rheault et al., (2001) quienes determinaron un alto porcentaje de resistencia frente a este antibiótico durante sus estudios a pesar de que se ha dejado de utilizar hace décadas en producción animal.

Finalmente, otro agente estudiado que presento sensibilidad intermedia fue el ácido nalidíxico, lo cual difiere de lo encontrado por Mejía (2003) quien encontró un porcentaje de resistencia del $8.3 \%$ de los aislamientos realizados durante su estudio frente a este antimicrobiano. Además por su amplio espectro y su alta potencia las quinolonas son consideradas como fármacos de primera elección en el tratamiento de infecciones producidas por bacterias gram negativas, por esta razón causa preocupación la aparición de cepas con sensibilidad reducida.

Por otro lado como resultado de los antibiogramas se evidenció una alta sensibilidad frente a la amoxicilina, un antimicrobiano utilizado comúnmente en el tratamiento de salmonelosis invasivas en humanos (Poirel et al., 1999). En algunos trabajos realizados se ha observado sensibilidad reducida frente a este compuesto (Espinasse et al., 1997; Grebeyes et al., 2000).
Posiblemente por la producción de betalactamasas originadas frente a betalactámicos más simples lo cual podría influir en su resistencia (Bradford et al., 1999; Fey et al., 2000).

Un agente antimicrobiano que demostró tener una elevada sensibilidad fue el cloranfenicol compuesto que se halla clasificado dentro de la familia de los anfenicoles, presentando diferencias significativas con lo expuesto por otros autores como Breuil et al., (2000), Martel et al., (2000) y Rheault et al., (2001) quienes encontraron niveles de resistencia muy altos para este producto a pesar de que su uso se ha restringido, dado sus efectos deletéreos sobre células eucarióticas (Vadillo, 2000).

Dentro de los resultados obtenidos se halló sensibilidad por parte de las cepas aisladas frente a kanamicina concordando con lo encontrado por Escobar (2004) quien encontró en un análisis de sensibilidad antimicrobiana realizadas a 10 cepas aisladas una alta sensibilidad frente a este compuesto, a su vez se encontró sensibilidad frente a enrofloxacina, una quinolona que por sus características de amplio espectro y eficacia son los antimicrobianos de primera elección ante cuadros clínicos producidos por bacterias gram negativas, como Salmonella. No obstante, se ha descrito la aparición de cepas de Salmonella con resistencia a este grupo de antimicrobianos (Hsih et al., 2004). La enrofloxacina ha sido descrita como uno de los antimicrobianos más utilizados en el tratamiento de problemas digestivos, por lo cual se hace hincapié en establecer el uso racional de la misma en los sistemas de producción, especialmente el porcícola, limitando la transferencia de resistencia entre cepas la cual limita la eficacia de estos antimicrobianos en casos clínicos de salmonelosis en humanos.

Por otra parte, la sensibilidad encontrada frente a la cefalexina coincide con lo encontrado por Zamora et al., (2006) quienes encontraron una sensibilidad del $82.5 \%$ frente a este compuesto en un estudio en el cual se analizó la sensibilidad de 40 cepas de Salmonella aisladas a partir de muestras de alimento. 
Frente a las sulfamidas, sulfametoxazoletrimetoprim, el presente estudio evidencio una alta sensibilidad en las cepas aisladas. No obstante, Fey et al., (2000), Walker et al., (2000), Chiu y Ou (2000), Weill (2008), así como Sisak et al., (2006) y Sisak et al., (2004) hallaron cepas de Salmonella resistentes a sulfamidas (Sulfametoxazole/ trimetoprim).

Los patrones de resistencia hallados en este trabajo difieren de lo reportado para las diferentes cepas de Salmonella en otras regiones geográficas del mundo (Grebeyes et al., 2000; Sisak et al., 2004; Sisak et al., 2006). Sin embargo, la caracterización molecular, la cual se encuentra en proceso, permitirá establecer diferencias moleculares precisas entre las cepas aisladas en el departamento del Tolima versus las aisladas en otras latitudes. Pinto et al., (2010) evaluaron molecularmente cepas de porcinos, las cuales difieren solo en la resistencia con las del presente

\section{REFERENCIAS}

Arroyo G, Arroyo JA. Detection of Salmonella serotypes in edible organ meats from markets in Madrid, Spain. Food Microbiol. 1995; 12:13-20.

Bauer A, Kirby W. Antibiotic susceptibility testing by a standardized single disk method. Am J Clin Pathol. 1966; 45(4):493-496.

Bradford PA, Petersen PJ, Fingerman IM, White DG. Characterization of expanded-spectrum cephalosporin resistance in $E$. coli isolates associated with bovine calf diarrhea disease. J Antimicrob Chemother. 1999; 44: 607-610.

Breuil J, Brisabois A, Casin I. Antibiotic resistances in salmonellae isolated from humans and animals in France: comparative data from 1994 and 1997. J Antimicrob Chemother. 2000; 46: 965-971

Carlson SA, Ferris KE. Augmentation of antibiotic resistance in Salmonella typhimurium DT104 following exposure to penicillin derivatives. Vet Microbiol. 2000; 73: 25-35 estudio en la presencia de resistencia al cloranfenicol, no obstante el análisis proteómico y de electroforesis 2-D, así como espectrometría de masa, no arrojó correlación entre la expresión de genes de resistencia y el comportamiento de la resistencia frente a los diferentes antibióticos.

En conclusión, la Salmonella se presenta como un microorganismo en producciones sin signología clínica evidente y aunque no induzca lesiones o cuadros clínicos representa un riesgo que pudiera influir subclinicamente en los parámetros productivos, más aun en procesos de inmunosupresión en la producción. La resistencia a antibióticos de las cepas aisladas permite establecer medidas de contingencia que incluyen la inclusión de terapia combinada de antibióticos y/o rotación de principios activos. Esto con el fin de disminuir la resistencia así como los estadios intermedios de la misma en los sistemas de producción.

Chiu C, Ou JT. Rapid identification of Salmonella serovars in feces by specific detection of virulence genes, invA and spvC, by an enrichment broth culture- multiplex PCR combination assay. J Clin Microbiol. 2000; 34: 2619-2622.

Degeeter MJ, Stahl GL, Geng S. Effect of lincomycin on prevalence, duration, and quantity of Salmonella typhimurium excreted by swine. Am J Vet Res. 1976; 37(5):525-9.

Espinasse F, Gheorghiu R, Poiata A, Labia R, Nicolas-Chanoine MH. Reduced susceptibility to co-amoxiclav in Escherichia coli, Salmonella Typhimurium and Klebsiella pneumoniae isolate in Romania between 1985 and 1993. J Antimicrob Chemother. 1997; 103-106.

FAO/OMS. Gestión de riesgos e inocuidad de los alimentos. Estudio FAO Alimentación y Nutrición -65. Roma, Italia, 1997. Fecha de consulta: 13 de febrero de 2007. 
Fey PD, Safranek TJ, Rupp ME, Dunne EF, Ribot E, Iwen PC, Brandford PA, Angulo FJ, Hinrichs SH. Ceftriaxone-resistant Salmonella infection acquired by a child from cattle. New England J Med. 2000; 342: $1280-1281$.

Flórez AC, Rincón C, Garzón P, Vargas N, Enríquez C. Factores relacionados con enfermedades transmitidas por alimentos en restaurantes de cinco ciudades de Colombia, 2007. Asociación Colombiana de Infectología 2008; 12(4): 255-266.

Gebreyes WA, Davies PR, Morrow WE, Funk JA, Altier C. Antimicrobial resistance of Salmonella isolates from swine. J Clin Microbiol. 2000; 38: 46334636.

Grebeyes WA, Davies PR, Morrow WEM, Funk JA, Altier C. Antimicrobial resistance of Salmonella isolates from swine. J Clin Microbiol. 2000; 38: 4633-4636.

Harakeh S, Yassine H, Gharios M, Barbour E, Hajjar S, El-Fadel M, Toufeili I, Tannous R. Isolation, molecular characterization and antimicrobial resistance patterns of Salmonella and Escherichia coli isolates from meat-based fast food in Lebanon. Sci Total Environment. 2005; 341(1-3): 33-44.

Hsih HY, Tsen HY. Combination of immunomagnetic separation and polymerase chain reaction for the simultaneous detection of Listeria monocytogenes and Salmonella spp in food samples. J Food Prot. 2004; 64: 1744-1750.

INS. Instituto Nacional de Salud. Sistema de Vigilancia Epidemiológica (SIVIGILA). Colombia, 2007. Fecha de consulta: 13 de febrero de 2008. uww.ins.gov.co

ISO 6579. Microbiology - General guidance on methods for the detection of Salmonella, International Organization for Standardization, Geneve, Switzerland. $4^{\text {rd }}$ ed. 2002.

Martel JL. Tardy F, Brisabois A, Lailler R, Courdert $M$. The French antibiotic resistance monitoring programs. Int J Antimicrob Agents. 2000; 14: 275-283.
Mejía W, Zapata D, Mateu E, Martin M. Lack of specificity of a combination of Rappaport-Vassiliadis broth and XLT4 agar for the isolation Salmonella form pig faeces. Vet Rec. 2005; 156:150-151.

NCCLS. CLSI/NCCLS Standard. Clinical and Laboratory Standards Institute. 2005.

Perez L. Estudio longitudinal de la salmonelosis en una granja porcina comercial. Trabajo de grado. Pontificia Universidad Javeriana. Bogotá, D.C. 2006.

Pinto L, Poeta P, Vieira S, Caleja C, Radhouani H, Carvalho $C$, Vieira-Pinto $M$, Themudo $P$, Torres $C$, Vitorino R, Domingues P, Igrejas G. Genomic and proteomic evaluation of antibiotic resistance in Salmonella strains. J Proteomics. 2010; 1535-1541.

Poirel L, Guibert M, Bellais S, Naas T, Nordmann $P$. Integrin- and carbanicellinase-mediated reduced susceptibility to amoxicillin- clavulanic acid in isolates of multidrug- resistant Salmonella enterica serotype Typhimurium DT104 from French patients. Antimicrobl Agents Chemother 1999; 1098-1104.

Quinn PJ, Markey BK, Carter ME, Donnely WJ, Leonard FC. Veterinary microbiology and microbial disease. Wiley-Blackwell, Blackwell Science. 2001; 114-118.

Rajik A, Keenliside J.Salmonella in swine. Advances in Pork Production. 2001; 12: 35-40.

Reid A. Isolation and identification of Salmonella from food and environmental samples. Health Products and Food Branch, Health Canada. Ottawa, Ontario. MFHPB20 - 2009.

Rheault N, Quessy S. Prevalence and resistance patterns of Salmonella spp. Serotypes from humans and production animals in Canada. In: Proceedings of the 4 International Symposium on the Epidemiology and Control of Salmonella and other borne Pathogens in Pork, Leipzig, Germany, 2001; 403-405.

Rondón-Barragán IS, Ramírez-Duarte WF, Gutiérrez G, Eslava Mocha PR. Edwardsiellosis 
en tilapia. In: XIII Jornada de Acuicultura, IALL Universidad de los Llanos, Septiembre 21 de 2007; 84-88.

Sisak F, Havlickova H, Hradecka H, Rychlik I, Kolackova I, Karpiskova R. Antibiotic resistance of Salmonella spp. isolates from pigs in the Czech Republic. Veterinarni Medicine. 2006; 5:303-310.

Sisak F, Havlickova H, Karpiskova R, Rychlik I. Prevalence of Salmonella and their resistance to antibiotics in slaughtered pigs in the Czech Republic. Czech J Food Sci. 2004; 22: 230-236.

Thakur YR, Bajaj BK. Antibiotic resistance and molecular characterization of poultry isolates of Salmonella by RAPD-PCR. World J Microbiol Biotech. 2006; 22(11): 1177-1183.

Thrusfield M. Veterinary epidemiology. $3^{\text {rd }}$ ed. Blackwell Science, Oxford, UK, 2007; 182-184.
Vadillo S. Manual de Microbiología Veterinaria. $2^{\circ}$ da Ed. Editorial Mc Graw Hill. Interamericana. 327-337 p.

Walker RA, Lawson AJ, Lindsay EA, Ward LD, Wright PA, Bolton FJ, Wareing DRA, Corkish JD, Davies RH, Threlfall EJ. 2000. Decreased susceptibility to ciprofloxacin in outbreak-associated multiresistant Samonella Typhimurium DT104. Vet Rec. 2000; 147:395-396.

Weill FX. Salmonella: épidémiologie, typage et résistance aux antibiotiques. Revue Francophone des Laboratoires. 2008; 400:37-37.

Zamora JM, Chaves C, Arias ML. Comparación del perfil de sensibilidad a antibióticos de cepas de Listeria monocytogenes y Salmonella spp. Aisladas a partir de alimentos con cepas de origen clínico. Facultad de Microbiología, Universidad de Costa Rica. Centro de Investigación en Enfermedades Tropicales. 2006; 56(2): 1-4. 